\title{
Frequency-dependent acoustic energy focusing in hexagonal ceramic micro-scaffolds
}

\author{
Tomáš Grabec ${ }^{\mathrm{a}}$, Martin Koller ${ }^{\mathrm{a}, *}$, Petr Sedlák ${ }^{\mathrm{b}}$, Alena Kruisová ${ }^{\mathrm{b}}$, \\ Benito Román-Manso ${ }^{c}$, Manuel Belmonte ${ }^{\mathrm{d}}$, Pilar Miranzo ${ }^{\mathrm{d}}$, Hanuš Seiner ${ }^{\mathrm{b}}$ \\ ${ }^{a}$ Faculty of Nuclear Sciences and Physical Engineering, Czech Technical University in \\ Prague, Trojanova 13, 12000 Praha 2, Czech Republic \\ ${ }^{b}$ Institute of Thermomechanics, Czech Academy of Sciences, Dolejškova 1402/5, 18200 \\ Praha 8, Czech Republic \\ ${ }^{c}$ School of Engineering and Applied Sciences, Harvard University, 52 Oxford Str, 02318 \\ Cambridge MA, USA \\ ${ }^{d}$ Institute of Ceramics and Glass (ICV-CSIC), Kelsen 5, 28049 Madrid, Spain
}

\begin{abstract}
Acoustic properties of a periodic hexagonal structure composed of thin rods are studied by finite element method (FEM) using Bloch boundary conditions. As a template for designing the computational unit cell, an additive-manufactured $\mathrm{SiC}$ scaffold fabricated by the robocasting method is used. The calculations show both angular and frequency dispersion of the acoustic waves with wavelengths comparable to the spacing between the rods, i.e., in a millimeter scale, indicating interesting acoustic properties in the $\mathrm{MHz}$ range. The dispersion character leads to focusing of the energy propagation into the directions of the rods of the hexagonal structure. This is illustrated by a modal-based calculation of the propagation of longitudinal and out-of-plane shear wave packets with a dominant wavelength.
\end{abstract}

Keywords: robocast scaffold, phononic crystal, acoustic energy focusing, Bloch analysis

\footnotetext{
${ }^{*}$ Corresponding author

Email address: martin.koller@fjfi.cvut.cz (Martin Koller)
} 


\section{Introduction}

Micro-architectured periodic structures often exhibit phononic-crystal behavior with acoustic phenomena taking place in the frequency ranges much lower than for any crystalline matter found in nature, such as frequency band 5 gaps in $\mathrm{MHz}$ range $[1,2,3,4,5$. Moreover, due to their highly ordered periodical arrangement, these structures are able to focus the acoustic waves along certain principal directions given by the geometry [6, 7, 8, 9].

With the rise of additive manufacturing (AM) methods, phononic crystals with various geometries can be easily produced [10, 11, 12]. Robocasting is a direct ink writing AM method, by which periodic structures consisting of layers of thin rods are printed from colloidal gel inks based on micro-sized powders [13, 14]. Silicon carbide ( $\mathrm{SiC})$ is often utilized for robocast scaffolds due to its favorable thermomechanical properties. Cai et al. [15] produced robocast scaffolds based on $\mathrm{SiC}$ with sintering additives of $\mathrm{Al}_{2} \mathrm{O}_{3}$ and $\mathrm{Y}_{2} \mathrm{O}_{3}$, utilizing pressure-less spark plasma sintering (SPS) for consolidation. The sintered scaffolds exhibited significant linear shrinkage when compared to green bodies, and the SPS lead to almost fully dense scaffolds with uniform microstructure while maintaining the geometric alignment of the rods. Román-Manso et al. [16] produced several SiC-based robocast scaffolds which differed in the mean size of the used $\mathrm{SiC}$ powders or in ratios between $\mathrm{SiC}$ and the sintering additives of $\mathrm{Al}_{2} \mathrm{O}_{3}$ and $\mathrm{Y}_{2} \mathrm{O}_{3}$. These scaffolds also preserved the designed geometry very well after the sintering and exhibited low porosity values and good indentation hardness. Furthermore, $\mathrm{SiC}$ was chosen as a material for support structures for electrically conductive robocast scaffolds with addition of graphene fillers [17], or for Fe-doped scaffolds for catalytic purposes [18.

Kruisová et al. [19] studied elastic and acoustic properties of the SiC-based scaffolds by resonant ultrasound spectroscopy (RUS) and finite element method (FEM) modeling. From the sintered scaffold, which had a perpendicular orientation of the rods in neighboring layers, a rectangular parallelepiped sample was cut. The elastic coefficients of this sample were then obtained by measur- 
ing free elastic vibrations by contactless RUS. The resonant spectrum of this sample consisted of sharp resonant peaks with high quality factor indicating low internal friction, suggesting that the rods are well-sintered and there is a good interlinking between the layers at the rods intersections. Due to the geome35 try, this scaffold macroscopically exhibits tetragonal symmetry. When applying FEM, it was shown that such a periodic scaffold is highly anisotropic in the tetragonal plane, which leads to the acoustic energy focusing along the ceramic rods. In the following works by Koller et al. 20, 21, acoustic properties of six types of scaffolds with three different types of material symmetry were studied.

40 It was shown that in tetragonal scaffolds, where the rods in neighboring layers are perpendicular, focusing of the acoustic energy along the rods is observed. Much stronger focusing occurs when the in-plane spacing between the rods is increased.

In another work of Kruisová et al. [22], transmission of ultrasonic waves 45 through four types of SiC-based scaffolds was studied. Longitudinal ultrasonic waves with frequencies varying from $2 \mathrm{MHz}$ to $12 \mathrm{MHz}$ were sent through the scaffolds in one of the directions of the ceramic rods, and the amplitudes of transmitted waves were measured. The wave propagation in these structures was also studied by FEM. It was shown both theoretically and experimentally that these so scaffolds, which have characteristic dimensions of 0.1-1 mm, exhibit phononiccrystal behavior with frequency band gaps in $\mathrm{MHz}$ range. This directly showed the interesting behavior of these structures - in standard crystals, phenomena such as the frequency band gaps can be observed in hardly reachable $\mathrm{THz}$ range.

In this paper, we investigate a scaffold with the orientation of the rods in neighboring layers of $60^{\circ}$ and the periodicity of 3 layers; thus, it exhibits macroscopic hexagonal symmetry. Since all directions in the hexagonal plane are macroscopically equivalent, no energy focusing was observed in the longwavelength limit. However, when the acoustic wavelength decreases and becomes comparable to the in-plane spacing between the rods, the energy becomes 6o focused along the rods, implying a frequency-dependent angular-dispersive character. A full FEM study of the acoustic behavior of a structure consisting of 
thousands of unit cells would be computationally very demanding. This problem was addressed by Hyun et al. [23], who developed an efficient and stable basis-vector-based model reduction scheme based on adaptive quasi-static Ritz

65 vector (AQSRV), and described its use for analysis and design of acoustic metamaterials. Similarly, a continuum model for the description of high-frequency behavior was developed by Rosi et al. 24] and tested on hexagonal and hexachiral structures [25]. However, in this paper, we propose a combination of FEM study of a unit cell with special, complex phase-shift boundary conditions in particular angles, followed by a simulation based on modal analysis.

\section{Materials and methods}

\subsection{Robocast structure}

The studied hexagonal scaffold structure, which is used as a template for designing the FEM unit cell, was fabricated by the robocasting technique. At first, a colloidal gel ink, based on $\beta$-SiC powder with sintering additives of $\mathrm{Al}_{2} \mathrm{O}_{3}$ and $\mathrm{Y}_{2} \mathrm{O}_{3}$, was produced by mechanical mixing of the powder mixture with ultrapure water and organic flocculants and viscosifiers. This is required because the ink needs to have highly shear-thinning rheological behavior, in order to form a thin filament when extruded through a nozzle tip following a

so computer-aided-design (CAD) printing route, and also to provide support for the already-printed free-standing structure. In each layer, the rods are mutually parallel with constant in-plane spacing between them, and the rods in the neighboring layers are oriented in a different angle of $60^{\circ}$ to form a hexagonal structure. The out-of-plane spacings between the layers are significantly lower than the diameters of the individual rods, producing scaffolds where the rods of neighboring layers partially intersect at crossing points. After the printing, the green bodies were dried in the air, the organic additives were then burnt out, and the scaffolds were subsequently consolidated by SPS.

The studied hexagonal scaffold structure, shown in Fig. 1. consists of a layered arrangement of the rods with diameter $d=215 \mu \mathrm{m}$, which are mutually 
parallel with constant in-plane spacing $l=576 \mu \mathrm{m}$ in each layer. The orientation angle between the rods in neighboring layers is equal to $60^{\circ}$ and the rods partially intersect at the crossing points with the out-of-plane spacing $z$ between the layers. This arrangement thus leads to a periodic hexagonal structure, having the out-of-plane periodicity of 3 layers, $h=3 z=385 \mu \mathrm{m}$.

(a)

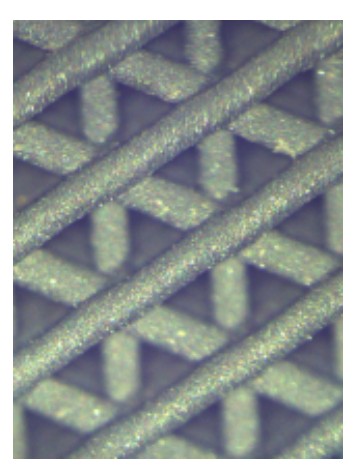

(b)

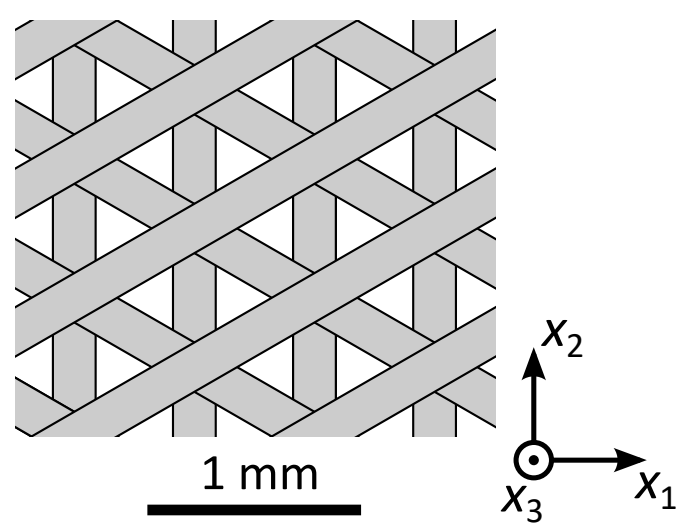

Figure 1: Top-view of the hexagonal structure, (a) optical micrographs of the consolidated scaffold, (b) geometric arrangement of the periodic structure.

(a)

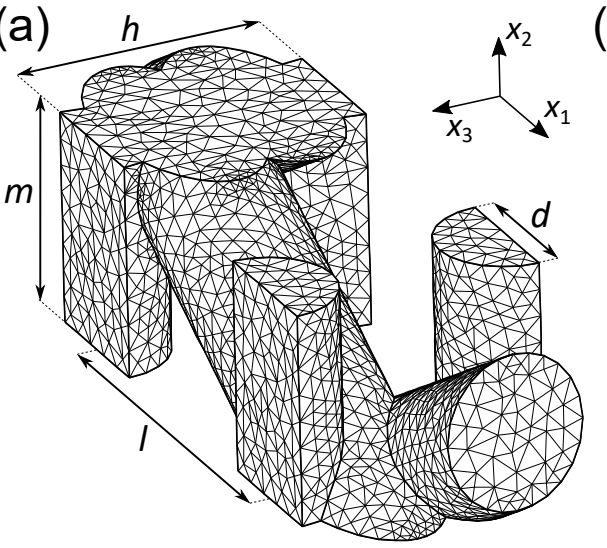

(b)

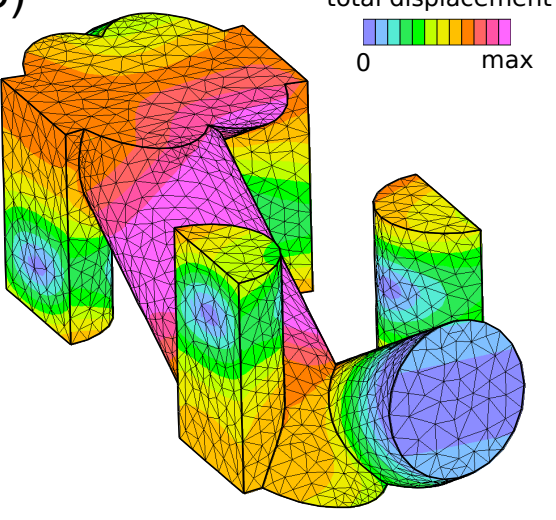

Figure 2: Computational unit cell used for FEM (a), the distance $m=(\sqrt{3} / 2) l$ is given by the geometry. The right side, (b), shows an illustrative example of the calculated displacement field for the propagation of a wave with high wavenumber $\left(k \approx 6.54 \mathrm{~mm}^{-1}\right)$ in the direction corresponding to $\alpha=20^{\circ}$. 


\subsection{FEM study}

Acoustic properties of the structure were studied by finite element method (FEM) using COMSOL Multiphysics software. A representative unit cell of the periodic structure was meshed with tetrahedral Lagrangian elements, as shown in Fig. 2(a). For the calculation, material properties of $\mathrm{SiC}$ were utilized [19]: a density of $3.34 \mathrm{~g} \cdot \mathrm{cm}^{-3}$, Young's modulus of $305 \mathrm{GPa}$, and Poisson's ratio of 0.19 . As the studied structure occupies a relative space of $44.4 \%$, the effective density is $1.483 \mathrm{~g} \cdot \mathrm{cm}^{-3}$. As described in [26], applying various types of periodic boundary conditions with prescribed displacement in the principal direction of the computation unit corresponds to macroscopic homogeneous straining of the structure and can be thus used for evaluation of macroscopic elastic coefficients of the hexagonal structure. The resulting constants obeying hexagonal symmetry for our structure are:

$$
c_{i j}=\left(\begin{array}{cccccc}
66.05 & 19.45 & 8.80 & 0 & 0 & 0 \\
19.45 & 66.05 & 8.80 & 0 & 0 & 0 \\
8.80 & 8.80 & 44.32 & 0 & 0 & 0 \\
0 & 0 & 0 & 9.30 & 0 & 0 \\
0 & 0 & 0 & 0 & 9.30 & 0 \\
0 & 0 & 0 & 0 & 0 & 23.30
\end{array}\right) \text { GPa. }
$$

Following the Bloch theorem [27, the wave propagation in the studied periodic structure can be sough in the form of planar waves with the displacement vector of

$$
\mathbf{u}(\mathbf{x}, t)=\mathbf{U}(\mathbf{x}, \mathbf{k}) \exp [\mathrm{i}(\mathbf{k} \cdot \mathbf{x}-\omega t)]
$$

where $\mathbf{k}$ is the wave vector. $\mathbf{U}(\mathbf{x}, \mathbf{k})$ is the Bloch waveform with the same spatial periodicity as the printed structure. Due to that, the computation domain can be limited to a single unit cell and the solution for different $\mathbf{k}$ is found by solution of eigenvalue problem with complex phase-shift boundary conditions, known as Bloch boundary conditions. In particular, to obtain wave dispersion of acoustic 
waves propagating in hexagonal plane, we applied the boundary conditions for rectangular supercell formed from 2 unit cells (see Fig 2 in the form

$$
\begin{aligned}
& \mathbf{u}\left(x_{1}=0, x_{2}, x_{3}\right)=\mathbf{u}\left(x_{1}=2 l, x_{2}, x_{3}\right) \exp \left(\mathrm{i} 2 l k_{x}\right), \\
& \mathbf{u}\left(x_{1}, x_{2}=0, x_{3}\right)=\mathbf{u}\left(x_{1}, x_{2}=2 m, x_{3}\right) \exp \left(\mathrm{i} 2 m k_{y}\right),
\end{aligned}
$$

and periodic boundary conditions in the direction of $x_{3}$ axis.

The wave solutions were determined for angles $\alpha$ (measured from the $x_{1}$ direction and following the notation given in Fig. 1) from $0^{\circ}$ to $30^{\circ}$ with $5^{\circ}$

steps. The direction of the ceramic rods is therefore equal to $\alpha=30^{\circ}$.

In each direction, the magnitude of $\mathbf{k}$ vector was increased from 0 to $4 \mathrm{~mm}^{-1}$ in 30 regular steps mapping approximately the inner two-thirds of the 1st Brillouin zone, which can be estimated as a circle with the radius $\pi / l$ in the hexagonal plane. The resulting dispersion curves for the longitudinal and slow shear wave, which has the polarization in the out-of-plane $\left(x_{3}\right)$ direction, are given in Fig. 3

\subsection{Modal analysis}

Figure 3 shows the results of the FEM simulation illustrating that with the increasing wavenumber, i.e., with the wavelength closer to the dimensions of the hexagonal structure, the wave propagation becomes gradually both angular- and frequency-dispersive.

To provide a simulation of wave propagation through a larger sample of the structure by a time-domain-type simulation, a very large model would be necessary, implicating high computational demands and time. Therefore, in order to calculate and visualize the propagation of wave-packets with different dominant wavenumber propagating through such a structure, we utilize a method based on modal characteristics with the following principle:

1. The dispersion curves obtained by FEM are fitted with a polynomial function in frequency and interpolated into the whole range of $\alpha$ angles applying the hexagonal symmetry. 
(a)

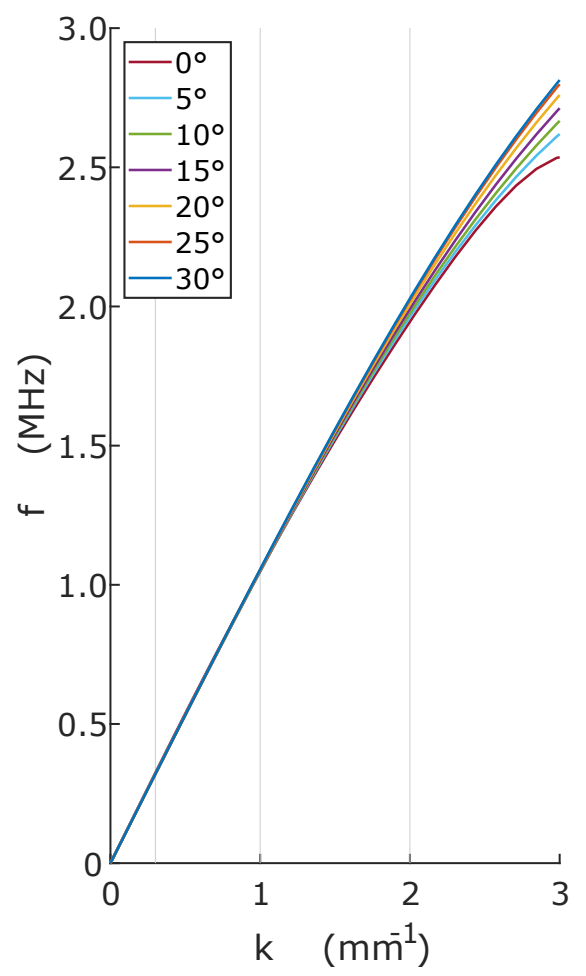

(b)

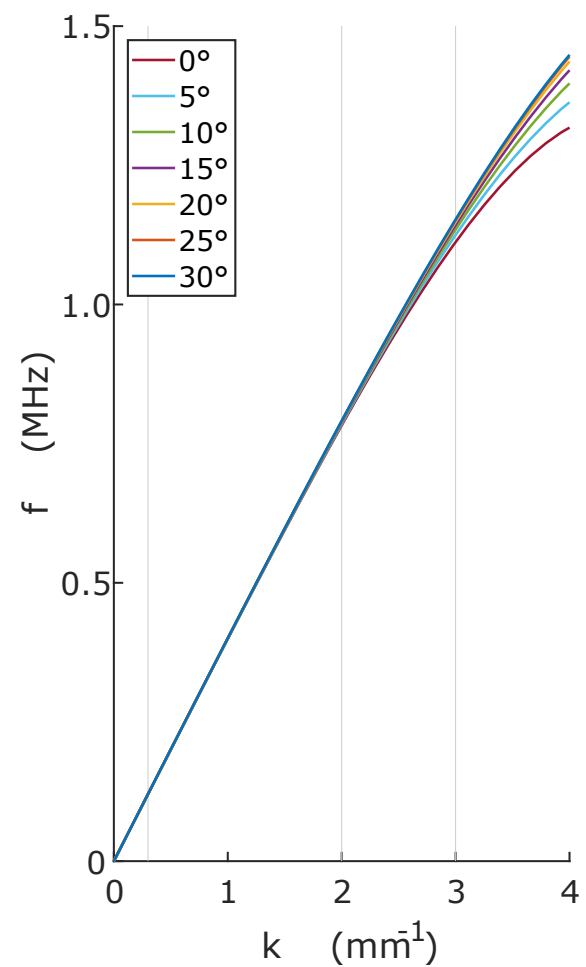

Figure 3: The dispersion data for the propagation of (a) longitudinal and (b) out-of-plane shear acoustic waves in the direction of the angle $\alpha$ with respect to $x_{1}$-axis, i.e., $\alpha=30^{\circ}$ corresponds to the direction of the rods. The curves are obtained via polynomial fit of the FEM data. The vertical lines correspond to wavenumbers chosen for the modal-simulations. 
2. The excitation is then modeled, given as an initial displacement field in the form of a centered radial cosine function superimposed by a Gaussian pulse, creating a spatial wave packet. Varying the wavelength of the cosine function, the dominant excited wavelength changes, while the

\section{Results and discussion}

Using the simulations based on modal analysis, the propagation characteristics of different wave packets through the described structure is studied.

Figure 4 shows the resulting displacement and intensity fields of longitudinalpolarized wave packets at time $t=10 \mu \mathrm{s}$. The chosen dominant wavenumbers correspond to the lines in Fig. 3(a), i.e., to the low-, middle- and highwavenumber region of the dispersion relation. It is illustrated that, whereas in the low-wavenumber, i.e., the long-wavelength region, the propagation shows no angular dispersion (see Fig. 4(a)), the influence of the structure increases with the shortening wavelength. In the middle region, the displacement field is still close to radial, but the intensity field shows obvious focusing of the energy propagation in the directions of the rods. In the high-wavenumber situation, the displacement is clearly hexagonal, Fig. 4(c). This is even more pronounced in the intensity, shown on the right side of Fig. 4(c), which indicates that the energy is in such a case transmitted almost exclusively through the rods. The 

larger than the in-plane rod spacing $l$.

In Fig. 5 a similar study for the case of out-of-plane shear waves at time $t=25 \mu \mathrm{s}$ is illustrated. Due to the larger dispersion (see Fig. 33(b)), the effect of focusing is even stronger. The lowest corresponding wavelength is about 3.5 larger than the rod spacing $l$.

Similarly to the case of band gaps at $\mathrm{MHz}$ range studied described in 22, in this case, the energy focusing corresponds to the frequencies around $1 \mathrm{MHz}$, as opposed to the frequencies in the range of $\mathrm{THz}$ needed for studying such effects in standard crystalline materials. This indicates not only interesting properties of the 3D printed structures but also offers a much easier way of studying the phenomena such as energy focusing in the far more easily-reachable frequency regions.

\section{Conclusion}

The precise 3D-printing technique of robocasting for creating structures of ceramic scaffolds offers a fast and simple way of constructing phononic crystals with interesting acoustic properties in the $\mathrm{MHz}$ range. In this paper, we studied the case of the scaffold with hexagonal symmetry.

With the model of the unit cell designed to mimic the printed structure, we obtained the dispersion curves of the acoustic modes in several directions by FEM calculations with Bloch boundary conditions (using the idea of complex phase shift at the boundaries). The resulting dispersion curves show increasing angular dispersion with higher frequency, i.e., with the corresponding wavelengths comparable to the in-plane rod spacing of the structure. As the spacing is equal to $576 \mu \mathrm{m}$, the dispersion is observable already at wavelengths of few millimeters (equivalent to frequencies around $1 \mathrm{MHz}$ in this case).

We studied this effect using the modal-based simulation of propagation of wave packets through a larger area of the hexagonal structure consisting of hundreds of cells. It can be seen that with a higher dominant frequency of the 

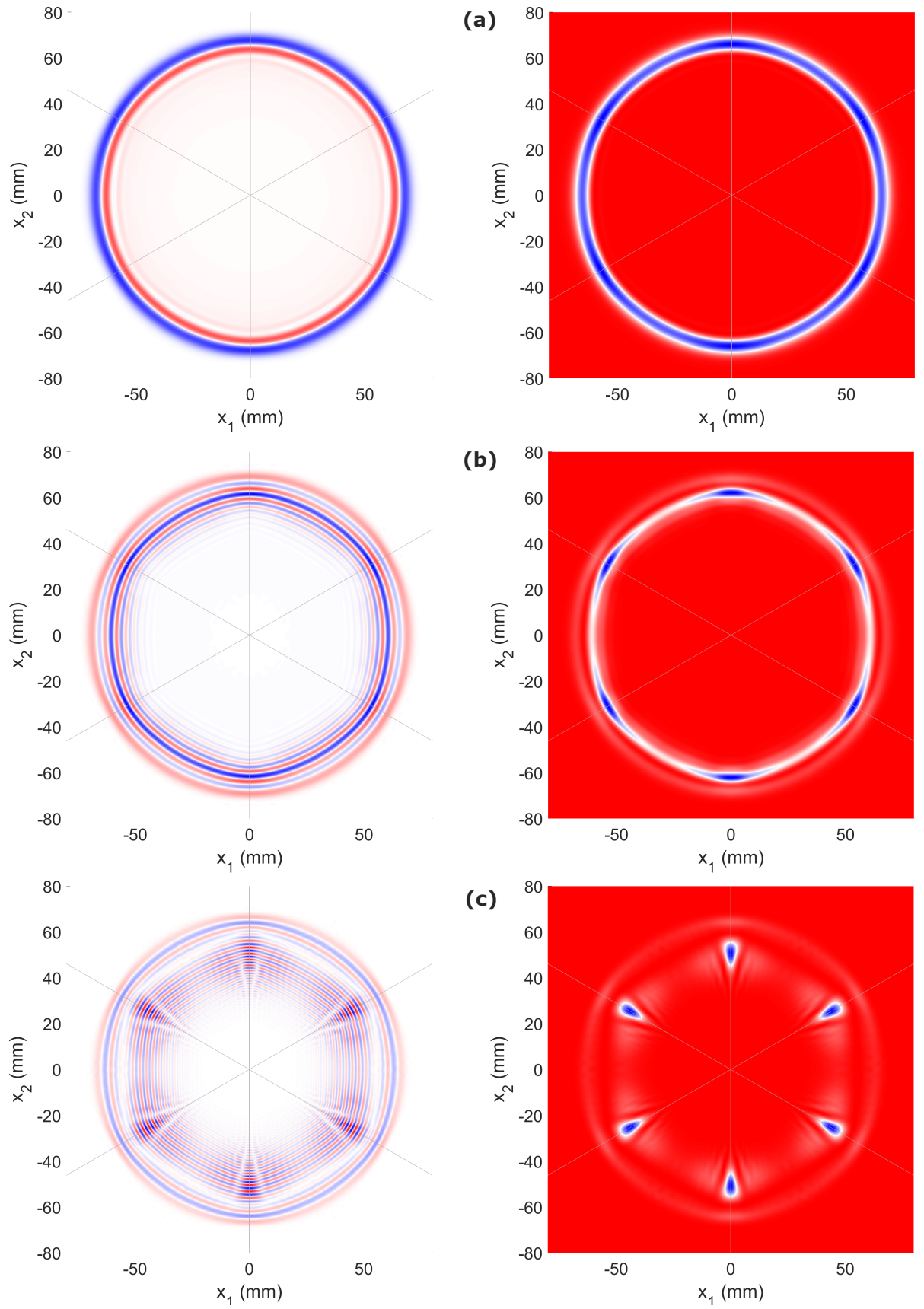

Figure 4: The displacement field (left) and the corresponding intensity (right) of longitudinal wave at $t=10 \mu \mathrm{s}$ for 3 different dominant wavenumbers: (a) $k=0.3 \mathrm{~mm}^{-1}$, (b) $k=1 \mathrm{~mm}^{-1}$, (c) and $k=2 \mathrm{~mm}^{-1}$. The light gray lines serve as the eye-guides of the angles under which the rods are oriented. 

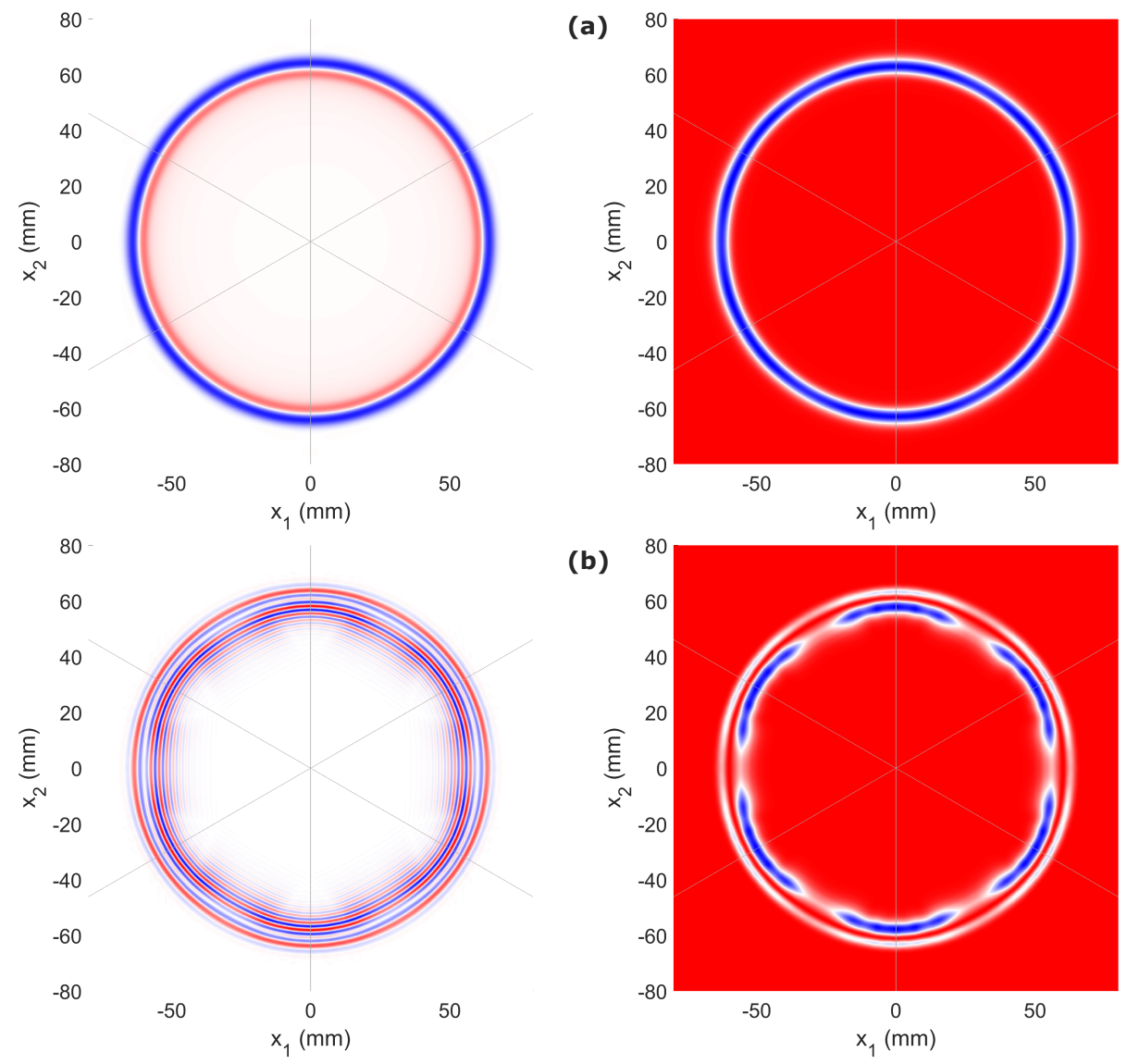

(b)
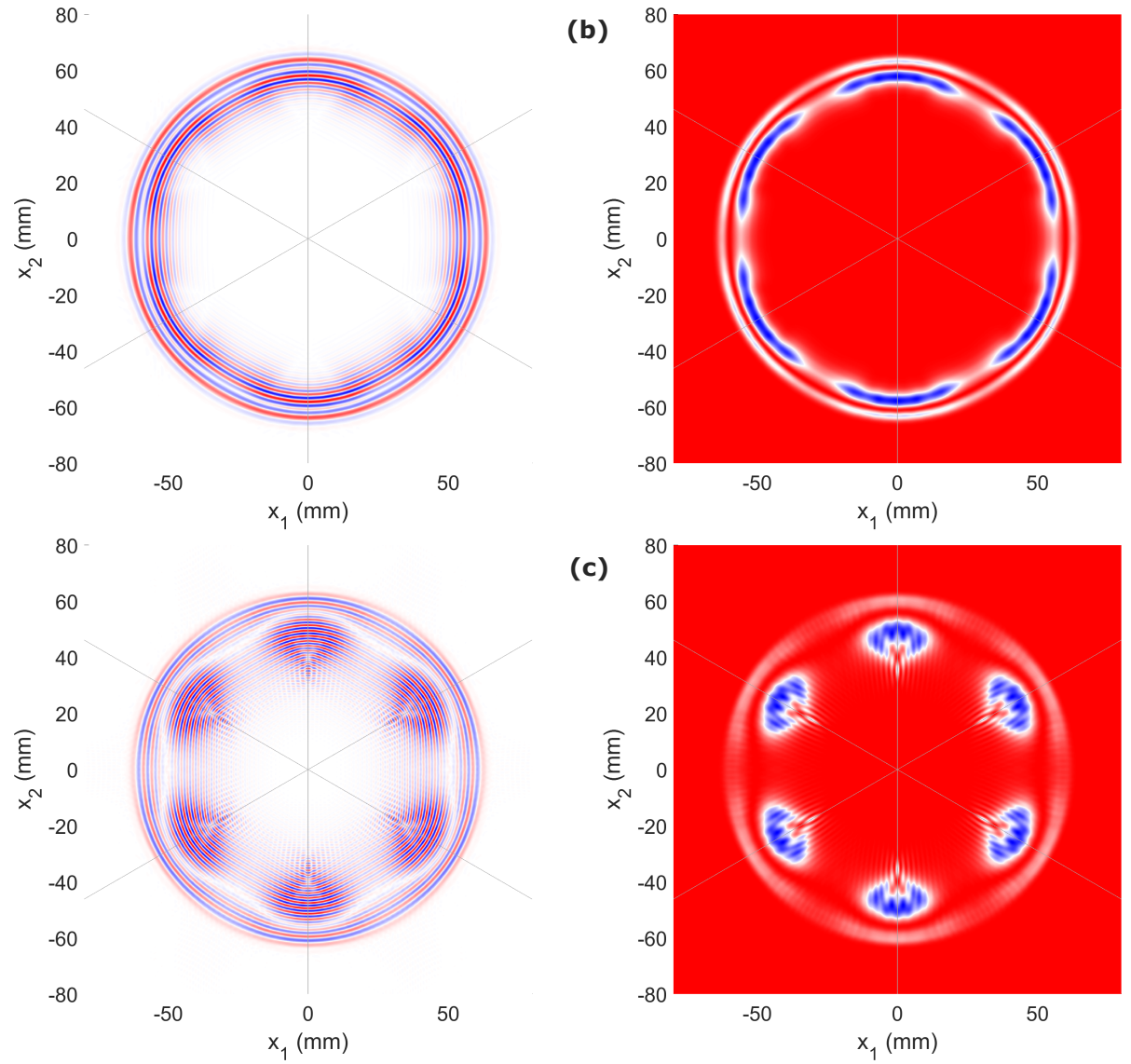

Figure 5: The displacement field (left) and the corresponding intensity (right) of out-of-plane shear wave at $t=25 \mu \mathrm{s}$ for 3 different dominant wavenumbers: (a) $k=0.3 \mathrm{~mm}^{-1}$, (b) $k=2 \mathrm{~mm}^{-1}$, (c) and $k=3 \mathrm{~mm}^{-1}$. The light gray lines serve as the eye-guides of the angles under which the rods are oriented. 
wave packet, the energy propagation is focused more into the direction of the rods. Already at frequencies around 1 or $2 \mathrm{MHz}$ (for shear or longitudinal waves, respectively), the energy propagates entirely through the rods. Corresponding to the previous study by Kruisová et al. [22, for higher frequencies, the group velocity is close to zero; hence, there is no energy propagation above a certain point, creating an acoustic band gap.

\section{Acknowledgement}

This work was supported by the Czech Science Foundation grant no. GA1701618S and by Spanish project MAT2015-67437-R (MINECO, FEDER, UE).

\section{References}

口 [1] M.-H. Lu, L. Feng, Y.-F. Chen, Phononic crystals and acoustic metamaterials, Materials Today 12 (12) (2009) 34-42. doi: 10.1016/S1369-7021(09)70315-3. URL http://www.sciencedirect.com/science/article/pii/ S1369702109703153

[ [2] Y.-Z. Wang, F.-M. Li, K. Kishimoto, Y.-S. Wang, W.-H. Huang, Elastic wave band gaps in magnetoelectroelastic phononic crystals, Wave Motion 46 (1) (2009) 47-56. doi:10.1016/j.wavemoti.2008.08.001

प URL http://www.sciencedirect.com/science/article/pii/ S0165212508000747

a [3] L. D'Alessandro, B. Bahr, L. Daniel, D. Weinstein, R. Ardito, Shape 200 optimization of solid-air porous phononic crystal slabs with widest full 3D bandgap for in-plane acoustic waves, Journal of Computational Physics 344 (2017) 465-484. doi:10.1016/j.jcp.2017.05.018.

a URL http://www.sciencedirect.com/science/article/pii/ S0021999117303935 
205 [4] Y. Chen, F. Meng, G. Sun, G. Li, X. Huang, Topological design of phononic crystals for unidirectional acoustic transmission, Journal of Sound and Vibration 410 (2017) 103-123. doi:10.1016/j.jsv.2017.08.015 URL http://www.sciencedirect.com/science/article/pii/ S0022460X1730617X

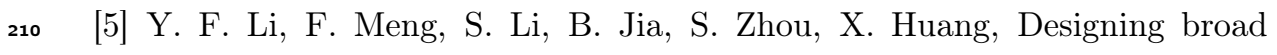
phononic band gaps for in-plane modes, Physics Letters A 382 (10) (2018) 679-684. doi:10.1016/j.physleta.2017.12.050.

घ URL http://www.sciencedirect.com/science/article/pii/ S0375960118300021

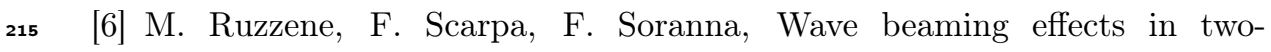
dimensional cellular structures, Smart Materials and Structures 12 (3) (2003) 363. doi:10.1088/0964-1726/12/3/307. URL http://stacks .iop.org/0964-1726/12/i=3/a=307

q [7] S. Yang, J. H. Page, Z. Liu, M. L. Cowan, C. T. Chan, P. Sheng, Focusing Honeycomb phononic crystals with self-similar hierarchy, Phys. Rev. B 92 230 (2015) 104304. doi:10.1103/PhysRevB.92.104304

URL https ://link. aps .org/doi/10.1103/PhysRevB.92.104304

[10] K. H. Matlack, A. Bauhofer, S. Krödel, A. Palermo, C. Daraio, Composite घ 3D-printed metastructures for low-frequency and broadband vibration ab- 
sorption, Proceedings of the National Academy of Sciences 113 (30) (2016)

8386-8390. doi:10.1073/pnas.1600171113

URL http://www.pnas.org/content/113/30/8386

[11] F. Lucklum, M. Vellekoop, Rapid prototyping of 3D phononic crystals using high-resolution stereolithography fabrication, Procedia Engineering $120 \quad$ (2015) 1095-1098, eurosensors 2015. doi:10.1016/j.proeng.2015.08.783.

口 URL http://www.sciencedirect.com/science/article/pii/ S1877705815024467

[12] T. Delpero, S. Schoenwald, A. Zemp, A. Bergamini, Structural engineering of three-dimensional phononic crystals, Journal of Sound and Vibration 363 (2016) 156-165. doi:10.1016/j.jsv.2015.10.033. URL http://www.sciencedirect.com/science/article/pii/ S0022460X1500869X

[13] J. Smay, G. Gratson, R. Shepherd, J. Cesarano, J. Lewis, Directed colloidal assembly of 3D periodic structures, Advanced Materials 14 (18)

250 ㅁ (2002) 1279-1283. doi:10.1002/1521-4095(20020916) 14:18<1279:: AID-ADMA1279>3.0.CO;2-A.

10 URL https://onlinelibrary.wiley.com/doi/abs/10.1002/

口. 1521-4095\%2820020916\%2914\%3A18\%3C1279\%3A\%3AAID-ADMA1279\%3E3. $0 . \mathrm{CO} \% 3 \mathrm{~B} 2-\mathrm{A}$

[14] J. Lewis, Direct ink writing of 3D functional materials, Advanced Functional Materials 16 (17) (2006) 2193-2204. doi:10.1002/adfm. 200600434

a URL https://onlinelibrary.wiley.com/doi/abs/10.1002/adfm. 200600434

[15] K. Cai, B. Román-Manso, J. E. Smay, J. Zhou, M. I. Osendi, M. Belmonte, 260 P. Miranzo, Geometrically complex silicon carbide structures fabricated by robocasting, Journal of the American Ceramic Society 95 (8) (2012) 2660-2666. doi:10.1111/j.1551-2916.2012.05276.x. 
URL https://onlinelibrary.wiley.com/doi/abs/10.1111/j. 1551-2916.2012.05276.x

[18] A. Quintanilla, J. Casas, P. Miranzo, M. Osendi, M. Belmonte, 3D-printed 1 Fe-doped silicon carbide monolithic catalysts for wet peroxide oxidation processes, Applied Catalysis B: Environmental 235 (2018) 246-255. doi:10.1016/j.apcatb.2018.04.066

口 URL http://ww.sciencedirect.com/science/article/pii/ S0926337318303953

[19] A. Kruisová, H. Seiner, P. Sedlák, M. Landa, B. Román-Manso, P. Mia ranzo, M. Belmonte, Acoustic metamaterial behavior of three-dimensional 285 periodic architectures assembled by robocasting, Applied Physics Letters 105 (21) (2014) 211904. doi:10.1063/1.4902810.

URL https://doi.org/10.1063/1.4902810

[20] M. Koller, A. Kruisova, H. Seiner, P. Sedlak, T. Grabec, B. Roman-Manso, a P. Miranzo, M. Belmonte, M. Landa, Ceramic phononic crystals with MHzrange frequency band gaps, Proceedings of Meetings on Acoustics 32 (1) 
(2017) 045005. doi:10.1121/2.0000690.

URL https://asa.scitation.org/doi/abs/10.1121/2.0000690

[21] M. Koller, A. Kruisová, H. Seiner, P. Sedlák, B. Román-Manso, P. Miranzo, M. Belmonte, M. Landa, Anisotropic elasticity of ceramic micro-scaffolds fabricated by robocasting, Acta Physica Polonica A 134 (3) (2018) 799-803. doi:10.12693/APhysPolA.134.799. URL http://przyrbwn.icm.edu.pl/APP/PDF/134/app134z3p42.pdf

[22] A. Kruisová, M. Ševčík, H. Seiner, P. Sedlák, B. Román-Manso, P. Miranzo, M. Belmonte, M. Landa, Ultrasonic bandgaps in 3Dprinted periodic ceramic microlattices, Ultrasonics 82 (2018) 91-100. doi:10.1016/j.ultras.2017.07.017.

1 URL http://www.sciencedirect.com/science/article/pii/ S0041624X17302408

[23] J. Hyun, J. Kook, S. Wang, Efficient and stable model reduction scheme for the numerical simulation of broadband acoustic metamaterials, Computers \& Mathematics with Applications 69 (8) (2015) 876-892. doi:10.1016/j.camwa.2015.01.004. URL http://www.sciencedirect.com/science/article/pii/ S0898122115000061

310 [24] G. Rosi, N. Auffray, Anisotropic and dispersive wave propagation within strain-gradient framework, Wave Motion 63 (2016) 120-134. doi:https://doi.org/10.1016/j.wavemoti.2016.01.009.

10 URL http://www.sciencedirect.com/science/article/pii/ S016521251600010X

315 [25] A. Spadoni, M. Ruzzene, S. Gonella, F. Scarpa, Phononic properties of hexagonal chiral lattices, Wave Motion 46 (7) (2009) 435-450. doi:10.1016/j.wavemoti.2009.04.002.

口 URL http://www.sciencedirect.com/science/article/pii/ S0165212509000341 
[26] A. Kruisová, H. Seiner, P. Sedlák, M. Landa, B. Román-Manso, P. Miranzo, M. Belmonte, Finite elements modeling of mechanical and acoustic properties of a ceramic metamaterial assembled by robocasting, in: Engineering Mechanics 2015, Vol. 821 of Applied Mechanics and Materials, Trans

a Tech Publications, 2016, pp. 364-371. doi:10.4028/www.scientific. 325 net/AMM. 821.364 .

[27] L. Brillouin, Wave propagation in periodic structures, 2nd Edition, Dover, 1953. 